\title{
Teletrabajo en las entidades públicas, una necesidad y prioridad en la actualidad
}

\author{
Andrés Avelino, Quispe Quispe \\ andresquispe9@gmail.com \\ ORCID: 0000-0003-2653-3094 \\ Escuela de Posgrado \\ Universidad César Vallejo \\ Lima - Perú
}

\author{
Giancarlo Andrés, Quispe Fernández \\ giancarloandres@gmail.com \\ ORCID: 0000-0003-1436-7551 \\ Escuela de Posgrado \\ Universidad César Vallejo \\ Lima - Perú
}

\section{RESUMEN}

El teletrabajo, hoy en día, se ha convertido en una modalidad de trabajo imprescindible para la continuidad de los servicios en las entidades públicas, encontrándose ligada a las personas, la tecnología y la regulación legal, propiciando la flexibilidad laboral de desempeño de actividades en un lugar distinto a la entidad. El objetivo es dar a conocer los aspectos más relevantes de su implementación, de su promoción y el impacto durante la pandemia del COVID-19. El método es teórico descriptivo de revisión documental, que consiste en la búsqueda, organización, sistematización y análisis de los documentos electrónicos seleccionados. Los resultados están basados en el uso de medios tecnológicos para el desarrollo de tareas y de guías laborales para el cumplimiento de los encargos planteados, ello, impactará y otorgará mayores beneficios a dichas organizaciones. Se concluye que, a nivel mundial, el sector público por la emergencia sanitaria, tuvo que implementar esta modalidad laboral, para los trabajadores, mediante la conectividad y las innovaciones tecnológicas, así como el uso de redes, plataformas y servicios digitales, obligadas a mantenerlas como una nueva forma de trabajo, en el contexto de la modernidad laboral.

Palabras clave: Entidades públicas, teletrabajo, trabajo remoto, trabajo a distancia. 


\title{
Teleworking in public entities, a need and priority today
}

\begin{abstract}
Teleworking, nowadays, has become an essential work modality for the continuity of services in public entities, being linked to people, technology and legal regulation, promoting labor flexibility in carrying out activities in a place other than the entity. The objective is to publicize the most relevant aspects of its implementation, its promotion and the impact during the COVID-19 pandemic. The method is a theoretical descriptive document review, which consists of the search, organization, systematization and analysis of the selected electronic documents. The results are based on the use of technological means for the development of tasks and labor guidelines for the fulfillment of the assignments, which will impact and grant greater benefits to said organizations. It is concluded that, worldwide, the public sector due to the health emergency, had to implement this work modality, for workers, through connectivity and technological innovations, as well as the use of networks, platforms and digital services, obliged to maintain them as a new way of working, in the context of modern labor.
\end{abstract}

Keywords: Public entities; telework; remote work; work from anywhere. 


\section{INTRODUCCIÓN}

El teletrabajo, mediante las nuevas tecnologías de la información y las comunicaciones (TIC) han cambiado la forma de trabajo y la vida diaria en el siglo XXI, pero, su uso para laborar fuera de las instalaciones de la entidad aún no se ha configurado en una praxis necesaria para todos los trabajadores (OIT y Eurofound, 2019); sin embargo, hoy en día, debido a la pandemia COVID - 19 que ha irrumpido intempestivamente la normalidad laboral, generando una crisis sanitaria a nivel global, recién las instituciones se han visto en la imperiosa necesidad de implementar el teletrabajo, con el fin de continuar con la prestación de los servicios de manera rápida, descentralizada y en ambientes remotos de trabajo. El presente estudio se estará abordando como problema la inoportuna implementación del teletrabajo en las entidades públicas.

Al respecto, Guillén (2021), señala que, en situaciones habituales, la cantidad de personas que teletrabajan en la Unión Europea, era el 10\% en República Checa, Italia, Polonia y Grecia; en Estados Unidos, aproximadamente el 20\%, 16\% en Japón. Asimismo, la OIT (2021), estimaba que en el 2019 había en el mundo unos 260 millones de personas asociadas al teletrabajo, que representaba solo el 7,9\% del empleo mundial. Y, Almonacid (2020), indica que las entidades arrastran insuficientes aspectos organizativos, que limitan el soporte a un manejo automático y electrónico. El teletrabajo, tuvo ciertas resistencias en su implementación hasta antes de la pandemia, se decían que no eran técnicamente posibles para su activación e implantación por aspectos de la tecnología de información y comunicaciones, cuando éstos son los que identifican a las instituciones modernas.

San Juan (2020), señala que en América Latina no existe un dispositivo legal regional o supranacional como si ocurre en el caso europeo, en el 2002 consiguió un acuerdo tripartito sobre el Teletrabajo. Además, refiere que antes de la crisis sanitaria, estaban normados en: Argentina, Brasil, Chile, Costa Rica, Colombia, Ecuador y Perú. Según Fernández y Bravo (2019), aseveran, a pesar que esta modalidad es una tendencia en aumento, las ciudadanías, con nuevos problemas y oportunidades, han respondido muy lento, con un resultado más por inercia tecnológica que por necesidades de los usuarios. Del mismo modo, Velásquez y Vera (2018), precisan, que no hay aceptación, por resistencia de las entidades y trabajadores, y que los directivos no están preparados para conducir al personal sino están en espacios de la entidad, así como, la falta de 
capacitación en manejo del teletrabajo, medio tecnológico y de organización, el personal tiene miedo al cambio, aislamiento, demora en las decisiones y subordinación de sus jefes.

Cortés (2017), precisa en el Perú, el teletrabajo se rige por la Ley $\mathrm{N}^{\circ} 30036$ y su reglamento el Decreto Supremo No 017-2015-TR, y por falta de digitalización en las instituciones, no se cuente con teletrabajadores, tampoco hay una convicción ni ánimo por parte de las entidades públicas, la necesidad de cubrir nuevas labores bajo esta modalidad de trabajo. Valencia (2018), manifiesta que, al contar con la política pública de gobierno electrónico, los funcionarios y servidores civiles deben valerse de los medios electrónicos para el cumplimiento de sus obligaciones y que se debe promover la cultura digital. Corresponde al Estado revisar las funciones, con el fin de establecer los puestos que puedan desarrollarse a través del teletrabajo, dada la tendencia en aumento de esta modalidad en el sector público.

Instituto Peruano de Economía (2020), advierte que en el 2019 tras seis años de vigencia de la ley de teletrabajo, solo 2,000 trabajadores estaban contratados en esta modalidad, representando el $0.01 \%$ de la fuerza laboral. Asimismo, el Banco Mundial identificó al Perú como el segundo país después de México y antes de Ecuador, donde los trabajadores presentan mayores dificultades para el teletrabajo. Delgado (2020), advierte que, por la crisis ocasionada por el virus COVID - 19, se emite el Decreto de Urgencia $\mathrm{N}^{\circ}$ 026-2020, para aplicación del trabajo remoto, una medida temporal que restringe el teletrabajo, que muy bien puede utilizarse para otros eventos de crisis en el futuro.

Yarnold (2021), indica, en cuanto a la aplicación del teletrabajo antes de la pandemia Covid-19, se generó un juicio limitado en las instituciones, se decía que, si en el centro laboral es difícil la supervisión, más complicado sería la supervisión a distancia, por ello, en el país no era atractiva. Existe una ley de teletrabajo, pero a nadie le interesaba su aplicación. El Comercio (2021), advierte, los servidores laboran presencialmente cuando pueden hacerlo en trabajo remoto, cuyo índice cayó de $33 \%$ entre abril y setiembre a $25 \%$ en último trimestre del 2020, dato reconocido por la entidad SERVIR; además, refiere que no todos los trabajadores cuentan con una conexión adecuada de Internet, necesariamente tienen que trabajar en las oficinas, y las entidades no pueden pagarles estos servicios, porque no están considerados en sus presupuestos, asociado a 
ello, el incumplimiento del aforo mínimo y respecto del personal considerado en grupo de riesgo haciendo labor presencial.

Este estudio tiene mucha importancia en la actualidad, porque la modalidad de teletrabajo es una modalidad legal para la atención de los servicios públicos, considerado como una estrategia generadora de flexibilidad para los trabajadores de realizar sus funciones fuera de las instalaciones de la entidad, ya diferentes países han revelado mucho interés en desplegar políticas de implementación de puestos laborales para que las personas utilicen herramientas tecnológicas para el desarrollo de sus actividades, lo cual es impulsado por los gobiernos a normarlo y promoverlo, coincidentemente afirman (Benjumea, Villa y Valencia, 2016; .Dapena, 2020; Tapasco y Giraldo, 2020; CLAD, 2020).

El estudio destaca significativamente que, esta modalidad es una realidad inevitable y que permanecerá en la sociedad en el tiempo y siempre que los trabajadores estén considerados dentro de los puestos teletrabajables, capacitándolos tecnológica y emocionalmente. Los medios de conexión digital, los avances en la calidad de las comunicaciones, el cambio de actitud de las instituciones y gestores que comienzan a notar el teletrabajo de manera favorable. Los gobiernos, debido a la emergencia sanitaria mundial, condujeron y estimularon a las instituciones a reconocer a sus trabajadores e instándoles a la utilización necesaria del teletrabajo, como enfatiza (Santillán, 2020).

Durante la pandemia provocada por el coronavirus, el contexto exige y el teletrabajo se transforma en una modalidad fundamental para la continuidad de las actividades institucionales, en los diferentes sectores durante el estado de emergencia sanitaria, esta manera de trabajo mejoró del $4 \%$ al $88 \%$ en las instituciones o sectores no esenciales y que no requieren de manera indispensable el modo presencial. La crisis sanitaria ha obligado cambios normativos frecuentes, principios y valores en las entidades, que ha significado adaptarse inmediatamente a ellos, en esta coyuntura, como señalan, (Suanzes, 2020; De La Torre, 2020; Roig y Pineda, 2020).

En este año, el teletrabajo en las entidades, se acentúa con más intensidad y es una de las modalidades de trabajo necesarios y prioritarios para prevenir los contagios, así como, una alternativa y oportunidad para la desconcentración de las labores y el desarrollo de competencias con el compromiso de convertirse en instituciones digitales; 
toda vez que, el teletrabajo incrementó significativamente el uso de Internet, medios digitales, el trabajo virtual y el uso de plataformas tecnológicas, en 324\%, el servicio Zoom o Google Meets en 75\%, tal como indica (Roncal, 2021).

El objetivo es dar a conocer los aspectos más relevantes de la implementación del teletrabajo en las entidades públicas que contratan teletrabajadores, las expectativas que se tiene con su promoción, así como las políticas públicas para su aplicación y la responsabilidad de desarrollo de las entidades del Estado, además identificar el impacto que ha tenido el teletrabajo en las personas que se encuentran laborando en esta modalidad producto de la pandemia por COVID-19 para la generación de estrategias institucionales.

\section{MÉTODOS}

El presente estudio es teórico descriptivo de revisión documental, que consiste en la búsqueda, organización, sistematización y análisis de documentos electrónicos sobre el teletrabajo en instituciones públicas, resultan de mucha importancia para determinar enfoques y nuevas áreas de estudio, empero también servirá para sintetizar y disponer de fundamentos sobre los cuales consolidar la base de una variable (Tramullas, 2020). Las unidades de análisis fueron todos aquellos documentos sobre el tema, encontrados en las plataformas o bases de datos en las que se efectuó la indagación en Scopus, Ebsco, Proquest, SciELO, Doaj, Dialnet, Latindex, Redalyc (Garcés y Stecher, 2021). Se tuvo como criterios de búsqueda, en 24 artículos de investigación científica, donde se incluyeron los siguientes descriptores o palabras clave: "teletrabajo", "trabajo remoto", "trabajo a distancia", “entidades públicas". Estos enunciados fueron combinados de diversas formas al momento de la exploración con el objeto de aumentar los criterios de rastreo de la variable (Crisol, Herrera y Montes, 2020). Al proceder la búsqueda de documentos, se priorizaron literaturas del período comprendido entre 2016 al 2021. Tipos de documentación encontrada en artículos científicos publicados en revistas open access, en el campo de las ciencias sociales, en las regiones de Latinoamérica y Europa, principalmente (Palacios y Deroncele, 2021). Finalmente, se ha procedido a realizar un análisis descriptivo, incidiendo en los resultados de cada publicación, a fin de encontrar elementos relevantes, comunes o divergentes. Para cada estudio se han tratado los datos correspondientes al período analizado, las técnicas de estudio aplicadas, las fuentes utilizadas, y los resultados 
obtenidos. Para este propósito se plantearon las siguientes interrogantes ¿Por qué no se implementó oportunamente el teletrabajo en las entidades públicas, antes de la pandemia? ¿Cómo fue la implementación del teletrabajo en las entidades públicas, durante la pandemia? ¿Qué efectos generará la pospandemia, en la implementación del teletrabajo en las entidades públicas?

\section{RESULTADOS}

Se describen los resultados de la revisión documental seleccionados, teniendo en cuenta las interrogantes planteadas, sobre la implementación del teletrabajo en las entidades públicas, según las siguientes tablas:

¿Por qué no se implementó oportunamente el teletrabajo en las entidades públicas antes de la pandemia?

\begin{tabular}{|c|c|c|}
\hline Autor (es) (año) & Nombre de artículo & Resultado \\
\hline Suárez (2016). & $\begin{array}{l}\text { Teletrabajo: realidad } \\
\text { y bienestar. }\end{array}$ & $\begin{array}{l}\text { Señaló, mayor flexibilidad, la persona puede } \\
\text { realizar su actividad laboral desde su domicilio } \\
\text { o desde el lugar donde se encuentre. Aumenta la } \\
\text { eficiencia y eficacia de las entidades públicas, } \\
\text { coadyuva en progreso de ciudades } \\
\text { interconectadas y sostenibles, mejora la calidad } \\
\text { de vida e impulsa el uso correcto de las TIC. }\end{array}$ \\
\hline $\begin{array}{l}\text { Guzmán y } \\
\text { Abreo (2017). }\end{array}$ & $\begin{array}{l}\text { Las habilidades del } \\
\text { teletrabajador para la } \\
\text { competitividad. }\end{array}$ & $\begin{array}{l}\text { Observaron, la falta de supervisión del jefe } \\
\text { inmediato sobre el subordinado, quien podría } \\
\text { tener un mayor tiempo de inactividad laboral, } \\
\text { incumplir normas de seguridad y salud en el } \\
\text { trabajo, pues la zona de labores podría no contar } \\
\text { con las condiciones mínimas requeridas para el } \\
\text { desempeño de la labor. Los teletrabajadores } \\
\text { obtienen un mayor compromiso con la entidad y } \\
\text { aceptan las actividades asignadas con mucha } \\
\text { responsabilidad, porque descubren un equilibrio } \\
\text { entre lo laboral y familiar, es decir, el teletrabajo } \\
\text { y el bienestar se encuentran estrechamente } \\
\text { relacionados como la contratación, el desarrollo } \\
\text { de laborales y las TIC. }\end{array}$ \\
\hline $\begin{array}{l}\text { Rolón y Sánchez } \\
\text { (2018). }\end{array}$ & $\begin{array}{l}\text { El teletrabajo: ¿una } \\
\text { opción laboral acorde } \\
\text { con las tendencias y } \\
\text { exigencias del } \\
\text { mercado o una figura } \\
\text { con vacíos } \\
\text { legislativos? }\end{array}$ & $\begin{array}{l}\text { Señalaron, un teletrabajador, viene a ser aquél } \\
\text { que realiza sus labores utilizando las TIC en otro } \\
\text { lugar ajeno a los locales de la entidad a la que } \\
\text { normalmente presta sus servicios, ello, dentro } \\
\text { del marco de la política pública de la promoción } \\
\text { del teletrabajo. }\end{array}$ \\
\hline Valle (2018). & $\begin{array}{l}\text { Teletrabajo, } \\
\text { necesidad y solución } \\
\text { en Méjico. Un estudio } \\
\text { exploratorio de sus } \\
\text { barreras culturales y } \\
\text { obstáculos. }\end{array}$ & $\begin{array}{l}\text { Determinó, es una manera flexible de esta } \\
\text { modalidad de trabajo, donde se realiza labores } \\
\text { sin la presencia física del trabajador en la } \\
\text { entidad durante la jorna laboral establecida. Es } \\
\text { un modelo de labor cada vez más aceptado } \\
\text { mundialmente, habiéndose implementado con }\end{array}$ \\
\hline
\end{tabular}




\begin{tabular}{|c|c|c|}
\hline & & $\begin{array}{l}\text { mucho éxito preferentemente en países de la } \\
\text { Unión Europea y Estados Unidos. }\end{array}$ \\
\hline \multirow[t]{2}{*}{$\begin{array}{l}\text { Telles y Spanier } \\
(2018) \text {. }\end{array}$} & $\begin{array}{l}\text { O teletrabalho: } \\
\text { conceituação e } \\
\text { questões para análise. }\end{array}$ & $\begin{array}{l}\text { Refirieron, las ventajas se asocian con la } \\
\text { flexibilidad del horario laboral, con la } \\
\text { organización del tiempo para conciliación } \\
\text { social, familiar, laboral y de entretenimiento. } \\
\text { Las desventajas se vinculan al impedimento de } \\
\text { supervisión y control sobre los trabajadores y la } \\
\text { desaparición de su integración y vinculación }\end{array}$ \\
\hline & & $\begin{array}{l}\text { con la entidad. Estos dos factores tienen una } \\
\text { fuerte unión entre sí, aludiéndose a la relación } \\
\text { que se establece entre el trabajador y la entidad. }\end{array}$ \\
\hline $\begin{array}{l}\text { Almonacid } \\
(2020) \text {. }\end{array}$ & $\begin{array}{ll}\text { El teletrabajo } & \text { en la } \\
\text { administración: } & \text { horas } \\
\text { eficientes } & \text { versus } \\
\text { horas de sillón (III). }\end{array}$ & $\begin{array}{l}\text { Precisó, la cultura de los trabajadores y, en } \\
\text { general de toda la entidad, fue el principal } \\
\text { obstáculo, porque no todas las personas poseen } \\
\text { el mismo perfil ni efectúan el mismo tipo de } \\
\text { actividades. El teletrabajo en una entidad } \\
\text { pública, en algunos puestos y con la instalación } \\
\text { apropiada, podría llevarse a cabo, no será al } \\
100 \% \text { de la jornada, pero sí de manera } \\
\text { considerable. El trabajo presencial es } \\
\text { justificable en pocos casos o en la mayor parte } \\
\text { de ellos, más por un tema formal que por } \\
\text { necesidad ineludible de la asistencia física. }\end{array}$ \\
\hline Buitrago (2020). & $\begin{array}{l}\text { Teletrabajo: una } \\
\text { oportunidad en } \\
\text { tiempos de crisis. }\end{array}$ & $\begin{array}{l}\text { Afirmó, las TIC no sustituyen al personal, son } \\
\text { medios útiles que facilitan ver la vida cotidiana } \\
\text { desde otra óptica, la eventualidad de } \\
\text { interconexión y el desempeño de labores sin la } \\
\text { presencia física en un lugar definido. Se necesita } \\
\text { un orden, estructura, determinación y capacidad } \\
\text { de adecuación al cambio, por parte de las } \\
\text { entidades y del personal. }\end{array}$ \\
\hline
\end{tabular}

Fuente: elaboración propia con base en los autores seleccionados.

¿Cómo fue la implementación del teletrabajo en las entidades públicas, durante la pandemia?

\begin{tabular}{|l|l|l|}
\hline Autor (es) (año) & Nombre de artículo & \multicolumn{1}{c|}{ Resultado } \\
\hline $\begin{array}{l}\text { Correa yoltrazio (2020). } \\
\text { Teletrabajo, formas de } \\
\text { organización, } \\
\text { problemas y desafíos. } \\
\text { Apuntes para aportar } \\
\text { al debate. }\end{array}$ & $\begin{array}{l}\text { Manifestaron, en la actual coyuntura, el papel } \\
\text { del Estado, de los servidores públicos y del } \\
\text { teletrabajo, son nuevas formas de establecer el } \\
\text { trabajo o nueva modalidad laboral, aplicables a } \\
\text { actividades múltiples, hace también concebirlo } \\
\text { como una modalidad y no como un trabajo, ya } \\
\text { que se da por una forma casual relacionada con } \\
\text { el espacio físico y la condición del personal. }\end{array}$ \\
\hline Ortega (2020). & $\begin{array}{l}\text { Por una cultura de } \\
\text { teletrabajo } \\
\text { emocionalmente } \\
\text { positivo } \\
\text { integralmente } \\
\text { saludable. }\end{array}$ & $\begin{array}{l}\text { Sentamos ante una oportunidad sin } \\
\text { precedentes de emitir normas laborales } \\
\text { debidamente conciliadas que permitan a las } \\
\text { personas ser más afables y saludables en este } \\
\text { naciente y extensivo mundo del trabajo. Las } \\
\text { reglamentaciones deben contener acciones de } \\
\text { traslado de labores a otros lugares y }\end{array}$ \\
\hline
\end{tabular}




\begin{tabular}{|c|c|c|}
\hline & & $\begin{array}{l}\text { capacitación funcional en domicilios, } \\
\text { flexibilización de jornadas respetando las horas } \\
\text { de trabajo, intervalo de descanso, compromiso } \\
\text { familiar, fijación de horas disponibles y } \\
\text { derecho a la desconexión digital. }\end{array}$ \\
\hline Ulate (2020). & $\begin{array}{l}\text { Teletrabajo y } \\
\text { impacto en la } \\
\text { productividad } \\
\text { empresarial y la } \\
\text { satisfacción laboral de }\end{array}$ & $\begin{array}{l}\text { Precisó, según estudios mundiales, el ahorro } \\
\text { por implementar el teletrabajo se logra hasta el } \\
45 \% \text {, demostrando ser una alternativa para el } \\
\text { uso apropiado de los recursos institucionales. } \\
\text { El } 73 \% \text { de las entidades consideraron que }\end{array}$ \\
\hline
\end{tabular}

\begin{tabular}{|c|c|c|}
\hline & $\begin{array}{l}\text { los colaboradores: } \\
\text { tendencias recientes. }\end{array}$ & $\begin{array}{l}\text { realizar labores en las oficinas en lugar de } \\
\text { aplicar la modalidad de teletrabajo } \\
\text { incrementaron sus costos. Otros advirtieron un } \\
\text { aumento del } 35 \% \text { de la efectividad de las } \\
\text { instituciones que emplearon el teletrabajo, } \\
\text { obteniendo satisfacción laboral, siempre que el } \\
\text { tele trabajador esté comprometido con su } \\
\text { institución. Considera, el teletrabajo afecta el } \\
\text { desempeño de los trabajadores, en este caso, las } \\
\text { instituciones, que han implementado el } \\
\text { teletrabajo, deben tener regulaciones sobre la } \\
\text { ética y calidad convenientes que les permitan } \\
\text { ejercer un estricto control sobre ellos. }\end{array}$ \\
\hline $\begin{array}{lll}\text { Peralta et al. } \\
(2020) .\end{array}$ & $\begin{array}{lll}\text { El impacto } & \text { del } \\
\text { teletrabajo } & \text { y } & \text { la } \\
\text { administración } & & \text { de } \\
\text { empresas. } & & \end{array}$ & $\begin{array}{l}\text { Indicaron, en relación a los nuevos enfoques } \\
\text { que se perciben en la era del conocimiento y de } \\
\text { la información, comportan la urgente necesidad } \\
\text { de abordar las modalidades de trabajo, políticas } \\
\text { para la disminución de la desocupación e } \\
\text { inclusión de los grupos vulnerables, nuevas } \\
\text { capacidades laborales, productividad, } \\
\text { innovación y la calidad de vida; además, la } \\
\text { implementación de esta modalidad, está ligada } \\
\text { a personas, tecnología y regulación legal, } \\
\text { pudiendo beneficiar a las entidades y a los } \\
\text { trabajadores, significa estar listos para esta } \\
\text { forma de trabajo, y, se cuente con las TIC que } \\
\text { permitan proteger datos y el acceso por } \\
\text { usuarios diferentes en un mismo momento, } \\
\text { permitiendo impactar y otorgar mayores } \\
\text { ventajas a las entidades públicas sobre aquellas } \\
\text { que aún mantienen trabajos convencionales. }\end{array}$ \\
\hline Morales (2020). & $\begin{array}{l}\text { Aspectos legales en } \\
\text { torno a la privacidad, } \\
\text { ciberseguridad } \\
\text { protección de datos } \\
\text { personales en el } \\
\text { Teletrabajo. }\end{array}$ & $\begin{array}{l}\text { Manifestó, será utilizada con mayor relevancia } \\
\text { una vez finalizada la pandemia COVID-19, } \\
\text { indudablemente es un adelanto importante en la } \\
\text { nueva forma de trabajo; sin embargo, debe } \\
\text { llegar equiparado con el acatamiento de la } \\
\text { normatividad regulatoria en diferentes } \\
\text { contextos, a fin de no inobservar los derechos } \\
\text { de los trabajadores y de las entidades en } \\
\text { "trabajo remoto" o "teletrabajo"; las entidades } \\
\text { están obligadas a cumplir con políticas de } \\
\text { seguridad de la información y proteger el }\end{array}$ \\
\hline
\end{tabular}




\begin{tabular}{|c|c|c|}
\hline & & derecho a la privacidad de las personas. \\
\hline $\begin{array}{l}\text { Tapasco y } \\
\text { Giraldo }(2020) .\end{array}$ & $\begin{array}{lrr}\text { Asociación } & & \text { entre } \\
\text { posturas } & & \\
\text { administrativas } & \text { de } \\
\text { directivos } & \text { y } & \text { su } \\
\text { disposición } & \text { hacia la } \\
\text { adopción } & & \text { del } \\
\text { Teletrabajo. } & & \end{array}$ & $\begin{array}{l}\text { Afirmaron, los directivos exponen como una de } \\
\text { las causas por la no utilización de esta } \\
\text { modalidad de trabajo, es el ámbito de } \\
\text { desconfianza por parte de las instituciones, } \\
\text { quienes expresan el miedo de consentirles a sus } \\
\text { subordinados a que laboren a distancia o desde } \\
\text { sus domicilios u otro lugar, lejanos a la } \\
\text { fiscalización de sus jefes inmediatos. }\end{array}$ \\
\hline
\end{tabular}

\begin{tabular}{|c|c|c|}
\hline Delgado (2020). & $\begin{array}{l}\text { El trabajo remoto en el } \\
\text { Perú en tiempos del } \\
\text { COVID- } 19 .\end{array}$ & $\begin{array}{l}\text { Indicó, es importante contar con normas } \\
\text { adecuadas para las entidades públicas donde se } \\
\text { promueva el teletrabajo ante cualquier tipo de } \\
\text { crisis sanitaria, u otras pandemias, a fin de tener } \\
\text { una herramienta útil de manera permanente. }\end{array}$ \\
\hline CEPAL (2020). & $\begin{array}{l}\text { Las oportunidades de } \\
\text { la digitalización en } \\
\text { América Latina frente } \\
\text { al Covid-19. }\end{array}$ & $\begin{array}{l}\text { Afirmó, en esta modalidad los trabajadores } \\
\text { usan plataformas virtuales para reuniones, } \\
\text { informar, coordinar y seguimiento de labores } \\
\text { con sus colegas y jefes. En este caso, no sólo se } \\
\text { trata de tecnología sino implica contar con un } \\
\text { marco legal que se regule para las instituciones } \\
\text { y establezca guías laborales del desempeño en } \\
\text { función de los encargos planteados. Esta } \\
\text { cultura organizacional no estaba desarrollada } \\
\text { en la mayoría de las entidades, conlleva desde } \\
\text { desafíos técnicos y normativos. El trabajo a } \\
\text { distancia puede ser una oportunidad de } \\
\text { modernizar el mundo laboral con cambio } \\
\text { estructural y la flexibilización del mercado } \\
\text { laboral. Es importante el acceso a Internet, } \\
\text { dispositivos y habilidades digitales. En } \\
\text { América Latina y el Caribe la era digital creció } \\
\text { más veloz que la provisión del talento humano. }\end{array}$ \\
\hline Yarnold (2021). & $\begin{array}{l}\text { La situación actual y el } \\
\text { futuro del teletrabajo } \\
\text { en el Perú. }\end{array}$ & $\begin{array}{l}\text { advirtió, según los indicadores que registró el } \\
\text { MTPE reflejó una cantidad reducida de } \\
\text { contratos en el sector público, recién a partir del } \\
2016 \text { se observaron algunos pilotos en las } \\
\text { entidades como: Indecopi, Osiptel, Ministerio } \\
\text { de Relaciones Exteriores y el Ministerio del } \\
\text { Ambiente. Los datos de incremento de } \\
\text { trabajadores en teletrabajo y en trabajo remoto } \\
\text { son: }(648) \text { en enero } 2017,(2,200) \text { en enero } \\
2020 \text { y }(220,000) \text { en julio } 2020 \text {. }\end{array}$ \\
\hline
\end{tabular}

Fuente: elaboración propia con base en los autores seleccionados.

¿Qué efectos generará la pos pandemia, en la implementación del teletrabajo en las entidades públicas?

\begin{tabular}{|l|l|l|}
\hline Autor (es) (año) & Nombre de artículo & \multicolumn{1}{c|}{ Resultado } \\
\hline Estévez y Solano & Tele Trabajo, Tele & Refieron, los aspectos positivos son el aumento \\
$(2021)$. & Emergencia, Tele & de la eficiencia laboral, la reducción del tiempo \\
& $\begin{array}{l}\text { Improvisación? } \\
\text { Análisis cualitativo a }\end{array}$ & $\begin{array}{l}\text { y los gastos por traslados, protección ambiental, } \\
\text { el mejoramiento de la satisfacción laboral, la }\end{array}$
\end{tabular}




\begin{tabular}{|l|l|l|} 
& $\begin{array}{l}\text { partir de experiencias } \\
\text { de informantes clave } \\
\text { en la administración } \\
\text { pública Argentina. }\end{array}$ & $\begin{array}{l}\text { disponibilidad de horarios libres, atención de } \\
\text { compromisos familiares. Las características } \\
\text { negativas, son el aislamiento laboral, perjuicio } \\
\text { en el desarrollo de la carrera y formación, menor } \\
\text { compromiso en el trabajo, exceso de horas } \\
\text { laboradas, riesgos físicos y psicológicos debido } \\
\text { al estrés y desconexión, mezcla de la vida } \\
\text { privada y deberes laborales. }\end{array}$ \\
\hline $\begin{array}{l}\text { García } \\
\text { Lafuente (2021). y }\end{array}$ & $\begin{array}{l}\text { El Estado en casa: Un } \\
\text { año de trabajo remoto. }\end{array}$ & $\begin{array}{l}\text { Expresaron, las instituciones públicas de todo el } \\
\text { mundo tuvieron que trasladarse por emergencia } \\
\text { al trabajo remoto, tuvieron inmediatamente que }\end{array}$ \\
\hline
\end{tabular}

\begin{tabular}{|l|l|l|}
\hline & & $\begin{array}{l}\text { adaptarse, gracias a los avances tecnológicos, } \\
\text { conectividad y la digitalización en la entidad y } \\
\text { trabajadores, una buena parte de ellos pudo } \\
\text { continuar con sus funciones desde sus } \\
\text { domicilios o de otros lugares. }\end{array}$ \\
\hline OIT (2021). & $\begin{array}{l}\text { Perspectivas sociales y } \\
\text { del empleo en el } \\
\text { mundo 2021 el papel } \\
\text { de las plataformas } \\
\text { digitales en la } \\
\text { transformación del } \\
\text { mundo del trabajo. }\end{array}$ & $\begin{array}{l}\text { Precisó, la era digital estácambiando el mundo } \\
\text { laboral. En la última década, se observa la } \\
\text { expansión de la conectividad, los servicios de } \\
\text { computación y las innovaciones en las } \\
\text { tecnologías de la información y las } \\
\text { comunicaciones. Prácticamente desde marzo de } \\
\text { 2020, la pandemia de la COVID-19 ha } \\
\text { ocasionado un aumento de las modalidades de } \\
\text { trabajo a distancia. }\end{array}$ \\
\hline $\begin{array}{l}\text { OIT - INAPI } \\
\text { (2021). }\end{array}$ & $\begin{array}{l}\text { Teletrabajo. } \\
\text { Modelo, manual y } \\
\text { recomendaciones } \\
\text { de política para INAPI. }\end{array}$ & $\begin{array}{l}\text { centro laboral, sino es una nueva forma de } \\
\text { trabajo, siendo un reto organizacional, manejo } \\
\text { tecnológico, localización, que requiere contar } \\
\text { con innovadoras políticas en gestión de talento } \\
\text { humano para el fortalecimiento del compromiso } \\
\text { yotivación laboral, en el contexto de los } \\
\text { deberes y desafíos de las instituciones públicas. }\end{array}$ \\
\hline
\end{tabular}

Fuente: elaboración propia con base en los autores seleccionados.

\section{DISCUSIÓN}

Para empezar, tal como manifiestan, (Guillén, 2021; OIT, 2021; San Juan, 2020), en épocas previas a la crisis sanitaria del 2020, a nivel mundial se tenía una cifra cercana al $8 \%$ de trabajadores en teletrabajo, en las naciones de la Unión Europea, EEUU y Japón, y con anterioridad, Valle (2018), indicaba del éxito de su implementación en el continente europeo y Estados Unidos; y en Latinoamérica, 7 países contaban con normas regulatorias de teletrabajo, entre ellos, el caso peruano. Sin embargo, según, el Instituto Peruano de Economía (2020), revelaba que, en Perú, los trabajadores en esta modalidad solo llegaban al escaso $0,1 \%$ y se ubicaba en segundo lugar entre los países de tener mayores problemas para el teletrabajo. 
También se dan a conocer, en cuanto a los obstáculos y limitaciones institucionales, según, Cortés (2017), el motivo por el que no se cuente con teletrabajadores es por la falta de digitalización en las entidades y, que en las instituciones no hay interés para cubrir nuevas plazas bajo esta modalidad de trabajo. Por su parte, Almonacid (2020), indicó, que las instituciones reflejan un insuficiente nivel organizativo, que restringen el uso digital, también señaló que, la cultura organizacional fue el principal obstáculo, porque no todo el personal tiene el mismo perfil ni ejecuta la misma labor, serían factibles para ciertos puestos con la correcta implementación, no cubrirá la jornada completa, pero sí para algunas horas. La posición anterior, es reforzada por (Valencia, 2018), afirmando que, corresponde a las entidades revisar las funciones y establecer los puestos teletrabajables. Y, según Bravo (2019), refirió, pese a que esta modalidad resulta ser una importante tendencia, se han respondido muy lento. Desde la óptica de (CEPAL, 2020; El Comercio, 2021), advirtieron, en la mayoría de las entidades, la cultura organizacional vinculada al teletrabajo no estuvo gestionada, conllevando a retos normativos y técnicos, así como, no todos los trabajadores contaban con una conexión a internet, aunado a ello, la falta presupuestal hizo que trabajaran necesariamente en modo presencial.

Se tiene otras dificultades atribuidas a la resistencia y aceptación, Guzmán y Abreo (2017), consideraron que, las entidades mostraban resistencia para aplicar esta modalidad, porque el jefe inmediato no supervisaba al subordinado, quien podría mantenerse más tiempo sin laborar, inobservar disposiciones de seguridad y salud en el trabajo, en el lugar donde laboraba. Asimismo, Velásquez y Vera (2018), aludieron que, los trabajadores y los directivos no estaban formados para gestionar personas fuera de las oficinas, además, indicaron la falta de capacitación en manejo del teletrabajo, en medios tecnológicos y de organización. Buitrago (2020), señaló que, se necesita un orden, estructura y capacidad de adaptación al cambio, de forma conjunta entre el personal y las instituciones. (Tapasco y Giraldo, 2020; Yarnold, 2021), enfatizaron que, uno de los motivos de que no se utilizara ni se diera importancia a esta modalidad de trabajo, fue la desconfianza de las entidades, porque la supervisión en el centro laboral es dificultosa y la vigilancia a distancia sería aún más compleja, incluso, el control de los jefes inmediatos, no estarían a su alcance. 
En cuanto a incumplimientos de protocolos sanitarios e implementación del teletrabajo, Morales (2020), mencionó que, es necesario cumplir las normas vigentes sobre el trabajo remoto o teletrabajo, en sus diversos ámbitos de aplicación, y evitar inobservancias de los derechos laborales de los trabajadores. Yarnold (2021), advirtió que, el número de trabajadores en teletrabajo a julio 2020, se incrementó sustantivamente en relación a periodos antes de la pandemia COVID-19; sin embargo, El Comercio (2021), reveló, que los servidores públicos vienen laborando en las instituciones de manera presencial, y que en el último trimestre del 2020, hubo una reducción significativa del trabajo remoto, además señaló que, se incumple el aforo mínimo establecido, y las personas del grupo de riesgo desempeñan labores en trabajo presencial, sin estar permitido ello.

En relación a la ventaja y desventaja, Guzmán y Abreo (2017), declararon que, el teletrabajo y la satisfacción laboral están ligados a la contratación, ejecución de tareas y uso de las TIC, y los teletrabajadores sentirán compromiso con la institución y cumplirán su labor con responsabilidad, al encontrar armonía de lo laboral y familiar. Telles y Spanier (2018), indicaron como ventaja la flexibilidad del horario laboral y como desventaja el dejar de lado la integración y su presencia física con la entidad. Por ello, Ulate (2020), propuso que, las entidades que implementen el teletrabajo, deben tener regulaciones sobre la ética y calidad, permitiéndoles controlar adecuadamente a las personas en esta modalidad. En esa línea, Estévez y Solano (2021), consideraron al teletrabajo, como positivo, por la mejora de la eficacia institucional, reducción de tiempo, disminución del gasto de movilidad, protección ambiental y horas libres; como negativo, al aislamiento laboral, perjuicio en la línea de carrera y capacitación, jornada extensa, riesgos físicos y psicológicos por causa del estrés y desconexión digital.

Respecto de su importancia antes de la pandemia, se consideraron como una modalidad necesaria para brindar servicios a la ciudadanía, que propiciaban la flexibilidad laboral en desempeñar funciones en lugar distinto de la entidad, los países mostraron su interés de implementar estratégicamente puestos de trabajo en esta modalidad, estableciendo normas para el progreso de ciudades interconectadas y sostenibles, (Suárez, 2016; Benjumea, Villa y Valencia, 2016; Dapena, 2020; Tapasco y Giraldo, 2020). Por otra parte, (Rolón y Sánchez, 2018; Peralta, et al., 2020), indicaron que, el teletrabajador, es aquél que desempeña actividades mediante las TIC, y que en la actualidad predomina el 
conocimiento e información, los mismos que son alineadas a las políticas laborales, competencias, efectividad, innovación y calidad de vida, con inclusión a grupos vulnerables. Delgado (2020) reiteró que, es indispensable que las entidades públicas tengan las normas adecuadas que fomenten el teletrabajo como una modalidad prioritaria y permanente ante cualquier evento de emergencia, que puedan suscitarse en el futuro.

Durante la pandemia adquiere mayor importancia, (Correa y Moltrazio, 2020; Santillán, 2020), coincidieron en precisar que, en actual circunstancia, el teletrabajo, resulta una excelente opción para el trabajo a distancia, aplicable a tareas diversas, a través de conexiones digitales, avances en comunicación, cambio de actitud en las instituciones y en el personal. Debido a la crisis generada por el COVID-19, los gobiernos establecieron el uso obligatorio de esta modalidad de trabajo, en reconocimiento a los trabajadores.

Mientras tanto, Delgado (2020), señaló que, se emitieron normas para aplicación del trabajo remoto, como una medida estrictamente temporal que limitó el teletrabajo, que podría servido para enfrentar otras dificultades posteriormente. Sin embargo, Ortega (2020), consideró que, las regulaciones estén orientadas especialmente a la flexibilización de la jornada laboral y el derecho a la desconexión digital. (Suanzes, 2020; De La Torre, 2020; Roig y Pineda, 2020), sostuvieron que, esta coyuntura ha obligado a las entidades a adaptarse a las disposiciones normativas y al cambio de cultura organizacional, mejorando sustancialmente el teletrabajo, en servicios no esenciales que no eran indispensables el trabajo presencial. Ulate (2020), indicó que, observó un incremento de la efectividad de las instituciones y una satisfacción laboral, por el compromiso de la tele trabajadora. Paralelamente, (CEPAL, 2020; Roncal, 2021), indicaron que, esta nueva forma de trabajo puede ser considerado como la oportunidad de modernizar el mundo laboral, por el incremento considerable del uso del trabajo virtual, uso de plataformas tecnológicas y el uso del servicio Zoom o Google Meets.

Que se espera después de la pandemia, según (García y Lafuente, 2021; OIT, 2021; OIT - INAPI, 2021), afirmaron que, desde el inicio de la pandemia COVID-19, el sector público a nivel mundial tuvo que adoptar el trabajo remoto por la situación de emergencia originada, de la mano con los avances de la conectividad digital y las innovaciones tecnologías de las TIC, capacitación a los trabajadores, implementadas por 
las entidades, precisando que estamos en la era digital que cambia el quehacer del mundo laboral. Además, es necesario se cuente con una política moderna en gestión de personas que refuercen la motivación y compromiso del personal en el sector público.

\section{CONCLUSIONES}

Desde un primer momento, el teletrabajo no fue implementado en las instituciones públicas, por falta de digitalización de sus procesos y trabajadores tampoco poseían un mismo perfil de puesto ni realizaban labores similares; entonces, corresponde a las entidades revisar y evaluar las funciones y, a su vez, identificar y determinar los puestos tele trabajables. Por esta situación se tuvo que enfrentar a transformaciones digitales y normas regulatorias, los trabajadores carecían de un adecuado acceso al Internet y las entidades no habían previsto en sus presupuestos para asumir estos gastos, sin otra opción, se vieron en la necesidad de realizar sus trabajos en modo presencial. Se podría decir que, la cultura organizacional respecto del teletrabajo, no estaba establecida. Los directivos carecían de competencias para dirigir al personal que realizan trabajo a distancia, quienes manifestaban lo difícil que es supervisar y controlar las actividades laborales en un lugar distinto al de la entidad; evidenciándose la inexistencia de un plan de capacitación en el manejo de esta modalidad de trabajo, uso de recursos tecnológicos y de la organización. Situaciones que impidieron la aplicación del teletrabajo.

Dada la implementación del teletrabajo o trabajo remoto, por efectos de la crisis sanitaria provocado por el COVID-19, según los datos reportados en el presente estudio, confirmarían que los trabajadores en vez de continuar laborando en modalidad remota lo estarían haciendo en modo presencial, disminuyendo así el número de tele trabajadores, incumpliéndose incluso los aforos sanitarios establecidos, es más, el personal considerado en grupo de riesgo, estarían laborando presencialmente, sin ser permitidos. Sería necesario acatar las disposiciones legales vigentes con la finalidad de respetar los derechos laborales de los trabajadores. Por tanto, el tele trabajador, es aquella persona que efectúa labores utilizando las TIC y en un lugar distinto al de la entidad. En este caso, las instituciones públicas, deben enfocarse en el desarrollo de competencias, eficiencia, eficacia y calidad de vida, que incluya a las personas vulnerables, y cuenten con normas legales sobre flexibilización de horarios y derecho de desconexión digital, a su vez, viabilicen el teletrabajo, como una modalidad de trabajo 
importante y permanente ante cualquier crisis que se generen en el futuro, asegurando así, la continuidad de los servicios que se prestan a la ciudadanía.

Finalmente, a nivel global, el sector público por la emergencia sanitaria, tuvo que implementar el teletrabajo o trabajo remoto, para los trabajadores, mediante la conectividad y las innovaciones tecnológicas, así como, el uso de redes, plataformas y servicios digitales, encontrándose ahora obligadas a mantenerlas como una nueva forma de trabajo, en este contexto de la modernidad laboral.

\section{REFERENCIAS}

Almonacid, V. (2020). El teletrabajo en la Administración: horas eficientes versus horas de sillón (III). Capital Humano, 351, 86-100. http://web.a.ebscohost.com/ehost/pdfviewer/pdfviewer?vid=23\&sid=bd97cfb0-d78d4959-a16b-f7ba450a39ca\%40sdc-v-sessmgr01

Almonacid, V. (2020). El coronavirus implanta el teletrabajo en el sector público o la constatación de que toda crisis es una oportunidad. Capital Humano, 352, 123-130.

http://web.a.ebscohost.com/ehost/pdfviewer/pdfviewer?vid=12\&sid=bd97cfb0-d78d-4959a16b-f7ba450a39ca\%40sdc-v-sessmgr01

Benjumea, M., Villa, M. y Valencia, J. (2016). Beneficios e impactos del teletrabajo en el talento humano. Resultados desde una revisión de literatura. Revista CEA, 2 (4), 5973. https://revistas.itm.edu.co/index.php/revista-cea/article/view/172/175

Buitrago, D. (2020). Editorial: “Teletrabajo: una oportunidad en tiempos de crisis".

Revista CES Derecho. 11(1), 1-2.

http://www.scielo.org.co/pdf/cesd/v11n1/2145-7719-cesd-11-01-1.pdf

Centro Latinoamericano de Administración para el Desarrollo - CLAD (2020).

Disposiciones normativas sobre el teletrabajo en los países CLAD como consecuencia de la Pandemia.

https://clad.org/wp-content/uploads/2020/05/Teletrabajo-PM-CLAD.pdf

CEPAL, (2020). Las oportunidades de la digitalización en América Latina frente al

Covid-19.

https://repositorio.cepal.org/bitstream/handle/11362/45360/4/OportDigitalizaCovid $\underline{-19 \_ \text {es.pdf }}$ 
Correa, F. y Moltrazio, C. (2020). Teletrabajo, formas de organización, problemas y desafíos. Apuntes para aportar al debate. Revista Derechos en Acción 5(16), 397-415 https://revistas.unlp.edu.ar/ReDeA/article/view/10689/9292

Cortés, J. (2017). Retos de la implementación del teletrabajo en el sector público peruano.

XXII Congreso Internacional del CLAD sobre la Reforma del Estado y de la

Administración Pública, Madrid, España.

file:///C:/Users/Sistema/Downloads/cortecar\%20servir.pdf

Crisol, E., Herrera, L. y Montes, R. (2020). Educación virtual para todos: una revisión sistemática. Education in the Knowledge Society. 21(15), 1-13. https://doi.org/10.14201/eks.20327

Dapena, M. (2020). Equipos de teletrabajo ágiles en las “Administraciones Públicas

remotas". Capital Humano, 358, 47-54.

http://web.a.ebscohost.com/ehost/pdfviewer/pdfviewer?vid=15\&sid=bd97cfb0-

d78d-4959-a16b-f7ba450a39ca\%40sdc-v-sessmgr01

De La Torre, C. (2020). El teletrabajo: nueva normativa y nueva realidad. ¿Una nueva relación entre empresa y empleado? Capital Humano, 357, 151-158.

http://web.a.ebscohost.com/ehost/pdfviewer/pdfviewer?vid=18\&sid=bd97cfb0-d78d-4959a16b-f7ba450a39ca\%40sdc-v-sessmgr01

Decreto de Urgencia $\mathrm{N}^{\circ}$ 026-2020. Decreto de urgencia que establece diversas medidas excepcionales y temporales para prevenir la propagación del coronavirus (covid-

19) en el territorio nacional. Diario Oficial El Peruano. https://busquedas.elperuano.pe/download/url/decreto-de-urgencia-que-establecediversas-medidas-excepcion-decreto-de-urgencia-n-026-2020-1864948-1

Decreto Supremo N ${ }^{\circ}$ 017-2015-TR. Decreto Supremo que aprueba el Reglamento de la Ley $N^{\circ}$ 30036, Ley que regula el teletrabajo. Diario Oficial El Peruano. https://busquedas.elperuano.pe/download/url/decreto-supremo-que-aprueba-elreglamento-de-la-ley-n-30036-decreto-supremo-n-009-2015-tr-1307067-3

Delgado, Á. (2020). El Trabajo Remoto en el Perú en Tiempos Del COVID-19. Revista Iberoamericana De Derecho Del Trabajo Y De La Seguridad Social, 2(3), 73-84. http://aidtss.org/revistaiberoamericana/index.php/main/article/view/34

El comercio, (2021). Trabajo remoto se usa cada vez menos en el sector público, mientras 
cuestionan protocolos en labor presencial.

https://elcomercio.pe/economia/peru/trabajo-remoto-se-usa-cada-vez-menos-en-el-

sector-publico-mientras-cuestionan-protocolos-en-labor-presencial-informe-

coronavirus-peru-noticia/

Estévez, A. y Solano, M. (2021). Tele Trabajo, ¿Tele Emergencia, Tele Improvisación? Análisis Cualitativo A Partir De Experiencias De Informantes Clave En La Administración Pública Argentina. Revista Ciencias Administrativas, 9(17), 65-78. http://www.scielo.org.ar/pdf/cadmin/n17/2314-3738-cadmin-17-64.pdf

Fernández, J. y Bravo, R. (2019). El Teletrabajo en América Latina: Derecho de Segunda y Cuarta Generación y de Ciudadanía Digital. Crítica Y Resistencias. Revista De $\begin{array}{llll}\text { Conflictos Sociales } \quad \text { Latinoamericanos, } & \text { (9), }\end{array}$ https://www.criticayresistencias.com.ar/revista/article/view/118

Garcés, M. y Stecher, A. (2021). El trabajo en tiempos de lean management: una revisión crítica sobre sus efectos adversos en las experiencias de trabajo. Innovar, 31 (79), 61-

78. $\quad$ https://doi.org/10.15446/innovar.v31n79.91889

García, M. y Lafuente, M. (2021). El Estado en casa: Un año de trabajo remoto. https://blogs.iadb.org/administracion-publica/es/el-estado-en-casa-un-ano-de-trabajoremoto/

Guillén, C. (2021). Cuidémonos. El teletrabajo ha llegado para quedarse. Gestión Práctica $\begin{array}{llll}\text { de Riesgos } & \text { Laborales, } & \text { 23-29. }\end{array}$ http://web.a.ebscohost.com/ehost/pdfviewer/pdfviewer?vid=9\&sid=bd97cfb0-d78d4959-a16b-f7ba450a39ca\%40sdc-v-sessmgr01

Guzmán, A. y Abreo, C. (2017). Las habilidades del teletrabajador para la competitividad.

Forum Empresarial, 22(2), 5-30.

https://www.redalyc.org/jatsRepo/631/63154910002/html/index.html

Instituto Peruano de Economía - IPE, (2020). Boletín IPE: ¿Trabajo remoto o teletrabajo? https://www.ipe.org.pe/portal/boletin-ipe-trabajo-a-distancia/

Ley $\mathrm{N}^{\circ}$ 30036. Ley que regula El Teletrabajo. Diario Oficial El Peruano. https://busquedas.elperuano.pe/normaslegales/ley-que-regula-el-teletrabajo-ley-n$\underline{\text { 30036-946195-3/ }}$

Morales, A. (2020). Aspectos Legales en torno a la privacidad, ciberseguridad y protección de datos personales en el Teletrabajo. http://agnitio.pe/articulo/aspectos-legales-en- 
torno-a-la-privacidad-ciberseguridad-y-proteccion-de-datos-personales-en-elteletrabajo/

OIT, (2021). Perspectivas Sociales y del Empleo en el Mundo 2021. El papel de las plataformas digitales en la transformación del mundo del trabajo. https://www.ilo.org/global/research/global-

reports/weso/2021/WCMS_771675/lang--es/index.htm

OIT y Eurofound, (2019). Trabajar en cualquier momento y en cualquier lugar:

consecuencias en el ámbito laboral.

https://www.ilo.org/wcmsp5/groups/public/---ed_protect/---protrav/---

travail/documents/publication/wcms_712531.pdf

OIT, (2021). El trabajo a domicilio de la invisibilidad al trabajo decente. https://www.ilo.org/global/publications/books/WCMS_765898/lang--es/index.htm

OIT (2021). Teletrabajo modelo, manual y recomendaciones de política para INAPI. https://www.inapi.cl/docs/default-source/2021/centro-dedocumentacion/estudios/teletrabajo/informe teletrabajo_inapi_oit_2021.pdf?sfvrsn $=1 \mathrm{~cd} 6873 \mathrm{~d} \_2$

Ortega, J. (2020). Por una cultura de teletrabajo emocionalmente positivo e integralmente saludable. Revista científica electrónica de Educación y Comunicación en la Sociedad del Conocimiento, 20(1), 1-3.

https://revistaseug.ugr.es/index.php/eticanet/article/view/15557

Palacios, M. y Deroncele, A. (2021). La Dimensión Socioemocional de la Competencia Digital en el marco de la Ciudadanía Global. Maestro Y Sociedad, 18(1), 119-131. https://maestroysociedad.uo.edu.cu/index.php/MyS/article/view/5318

Peralta, A., Bilous, A. Flores, C. y Bombón, C. (2020). El impacto del teletrabajo y la

administración de empresas. RECIMUNDO, 4(1), 326-335. https://recimundo.com/index.php/es/article/view/761/1154

Roig, R. y Pineda, C. (2020). El teletrabajo y la conciliación: dos políticas públicas diferentes. GIGAPP Estudios Working Papers, 7(187), 593-608. http://www.gigapp.org/ewp/index.php/GIGAPP-EWP/article/view/203/236 
Rolón, F. y Sánchez, S. (2018). El teletrabajo: ¿una opción laboral acorde con las tendencias y exigencias del mercado o una figura con vacíos legislativos?. Dictamen Libre, 1(22), 91-104. https://doi.org/10.18041/2619-4244/d1.22.5030

Roncal, X. (2021). Teletrabajo y Capitalismo de Vigilancia. Telos: revista de Estudios Interdisciplinarios en Ciencias Sociales, 23(1), 177-192. www.doi.org/10.36390/telos231.14

San Juan, C. (2020). Estudio comparado de la legislación sobre Teletrabajo en los países

de América Latina y el Caribe.

http://www.relats.org/documentos/SST.ALC.General.SanJuanJulio2020.pdf

Santillán, W. (2020). El Teletrabajo en el COVID-19. CienciAmérica, 9(2), 65-76. http://dx.doi.org/10.33210/ca.v9i2.289

Suanzes, J. (2020). Teletrabajo: pandemia y nueva regulación. Capital Humano, 359, 3-9. http://web.a.ebscohost.com/ehost/pdfviewer/pdfviewer?vid=7\&sid=bd97cfb0-d78d4959-a16b-f7ba450a39ca\%40sdc-v-sessmgr01

Suárez, A. (2016). Teletrabajo: realidad y bienestar. Revista de Investigaciones de la Escuela de Ciencias Sociales, Artes y Humanidades - UNAD, 7, 17-32.

file:///C:/Users/Sistema/Downloads/2299-Texto\%20del\%20art\%C3\%ADculo-6554-1-1020180425\%20(3).pdf

Tapasco, O. y Giraldo, J. (2020). Asociación entre Posturas Administrativas de Directivos

y su Disposición Hacia la Adopción del Teletrabajo. Información tecnológica, 31(1), 149-160.

file:///C:/Users/Sistema/Downloads/20085.09_Posturas_hacia_la_adopcion_del_tel

etrabajo.pdf

Telles, C. y Spanier, F. (2018). O teletrabalho: conceituação e questões para análise. Cadernos EBAPE.BR, 16(1), 152-162. https://www.redalyc.org/articulo.oa?id=323257350011

Tramullas, J. (2020). Temas y métodos de investigación en Ciencia de la Información, 2000-2019. Revisión bibliográfica. Profesional de la información, 29(4). https://doi.org/10.3145/epi.2020.jul.17

Ulate, R. (2020). Teletrabajo y su Impacto en la Productividad Empresarial y la Satisfacción Laboral de los Colaboradores: Tendencias Recientes. Revista Tecnología En Marcha, 33(7), 23-31. https://doi.org/10.18845/tm.v33i7.5477

Valencia, A. (2018). Aspectos regulatorios del teletrabajo en el Perú: análisis y 
perspectivas. Revista IUS, 12(41), 203-226.

http://www.scielo.org.mx/scielo.php?script=sci_arttext\&pid=S1870-

21472018000100203\&lng=es\&tlng=es.

Valle, G. (2018). Teletrabajo, necesidad y solución en Méjico Un estudio exploratorio de sus barreras culturales y obstáculos. TEUKEN BIDIKAY Revista Latinoamericana de Investigación en Organizaciones, Ambiente y Sociedad, 9(12), 153-176. https://doaj.org/article/8e04970f8fac43bfa1953f8d161686b0

Velásquez, C. y Vera, M. (2018). Teletrabajo: Una Revisión Teórica sobre sus Ventajas

y Desventajas. INVESTIGATIO, 10, 41-53.

https://revistas.uees.edu.ec/index.php/IRR/article/view/194/123

Yarnold, E. (2021). La situación actual y el futuro del teletrabajo en el Perú. http://www.cielolaboral.com/la-situacion-actual-y-el-futuro-del-teletrabajo-en-el-peru/ 